\title{
Dermatoneuro syndrome
}

\section{A full recovery after a second episode}

Andrew Liu, MD; Kathleen Suozzi, MD; David Y. Hwang, MD; Jeremy J. Moeller, MD; Rossitza Lazova, MD;

Daniel DiCapua, MD

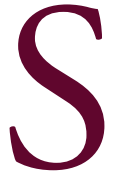

cleromyxedema is a chronic, idiopathic disorder associated with monoclonal gammopathy and characterized by dermal mucin deposition. ${ }^{1} \mathrm{~A}$ rare but severe complication of scleromyxedema is dermatoneuro syndrome (DNS), which presents with a flu-like prodrome, skin papules, fever, seizures, and coma. It can have catastrophic systemic effects with a substantial mortality rate of $21 \%^{2,3}$ requiring a multidisciplinary diagnostic approach for early detection and management.

\section{Case Report}

A 59-year-old woman with a history of scleromyxedema complicated by DNS 15 years previously and remote history of breast cancer presented to the emergency department (ED) with incoherent speech.

The patient was in her normal state of health until she developed fever, malaise, and myalgia after an IV immunoglobulin (IVIg) infusion, which she had received every 3 weeks since her first episode of DNS. Four days later, a family member noted incoherent speech and brought the patient to the ED. She was admitted to the general neurology service.

Initial neuroimaging was unremarkable for acute intracranial pathology. On physical examination, firm waxy papules were noted on dorsolateral hands, posterior neck, and ears, stable from previous examinations. The patient's mental status deteriorated along with progression to global aphasia. She developed tachypnea, hypotension requiring fluid resuscitation, and atrial fibrillation with heart rates between 140 and 160, which responded to metoprolol. This dysautonomia continued for 2 days. Blood cultures, urinalysis, urine culture, CSF analysis, and imaging of chest, abdomen, and pelvis were all unrevealing for infectious, metabolic, or neoplastic etiologies.

The patient's clinical course up to this point was similar to her prior episode of DNS, leading the neurology team to suspect a recurrence. The initial diagnosis of scleromyxedema was based on a skin biopsy from 2000 (figure 1). Several months later, the patient presented with seizures and a fever of $104^{\circ} \mathrm{F}$. The dermatologist believed her presentation was consistent with DNS and she was treated with the same immune modifiers. Dermatology was consulted and agreed with the diagnosis of DNS. Treatment was initiated on hospital day 2 with methylprednisolone $1 \mathrm{~g}$ IV for 5 days, plasma exchange every other day for 10 days, and subsequent IVIg $0.4 \mathrm{mg} / \mathrm{kg} / \mathrm{d}$ over 5 days.

On hospital day 4, the patient became comatose and was transferred to the neuroscience intensive care unit following intubation. CT head without contrast showed mild asymmetry of the hippocampi, possibly representing artifact or subtle postictal sequela. On hospital day 6 , the patient was found to be in nonconvulsive status epilepticus by continuous EEG monitoring (figure 2). She required 3 antiepileptic drugs for seizure control, including valproic acid, levetiracetam, and lacosamide. The patient was subsequently extubated, regained baseline mental status, and was discharged to short-term rehabilitation after a 20-day hospital

\section{Practical Implications}

Dermatoneuro syndrome, a rare complication of

scleromyxedema associated with skin lesions, seizures,

and coma, requires a multidisciplinary approach to diagnosis and management.

Andrew Liu, Yale-New Haven Hospital, Departments of Neurology (AL, DYH, JJM, DD), Dermatology (DYH), and Pathology (RL), Yale-New Haven Hospital, CT.

Funding information and disclosures are provided at the end of the article. Full disclosure form information provided by the authors is available with the full text of this article at Neurology.org/cp.

Correspondence to: Andrew.liu@yale.edu 
Figure 1 Dermatopathology
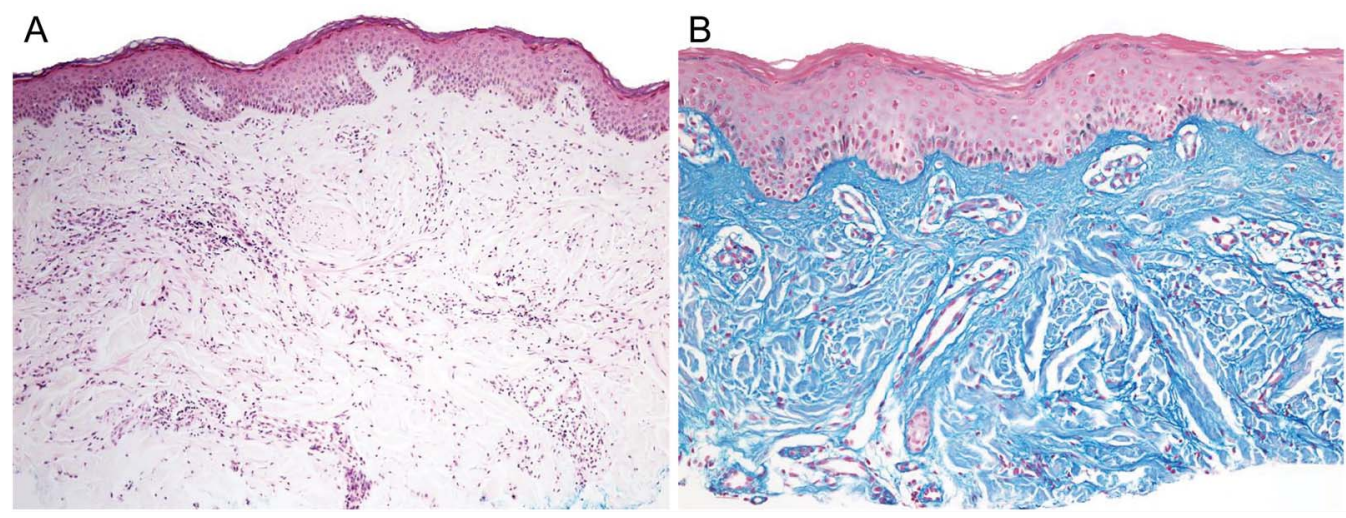

(A) The dermis is cellular with increased numbers of fibroblasts in the interstitium. The spaces between collagen bundles are slightly widened. In addition, there is a perivascular lymphocytic inflammatory infiltrate. (B) There is an increased amount of mucin in the dermis, highlighted by a colloidal iron stain.

course. Antiepileptic medications were tapered off with EEGs showing no epileptiform activity. The patient restarted maintenance dose IVIg $40 \mathrm{~g}$ every 21 days. She is now able to perform normal activities of daily life, but remains amnestic to her hospitalization.

\section{DISCUSSION}

Scleromyxedema is an idiopathic disorder characterized by numerous firm papules with areas of skin induration caused by mucin deposition and fibroblast proliferation with fibrosis and is nearly always associated with a plasma cell dyscrasia, most commonly an immunoglobulin G (IgG) $\lambda$ gammopathy (figure $1, \mathrm{~A}$ and B). ${ }^{1,2,4}$ The role of the paraproteinemia in the pathogenesis of the disease is unknown. Rarely, patients can have severe systemic symptoms including neurologic, rheumatologic, cardiovascular, renal, and pulmonary complications. The constellation of flu-like prodrome, fever, seizures, and CNS disturbances leading to coma is termed DNS and is a rare, life-threatening complication. ${ }^{1,5}$

The management of DNS requires a multidisciplinary approach, including early recognition of altered mental status in the ED. The mortality rate based on previously reported cases is $21 \%$

Figure 2 Imaging

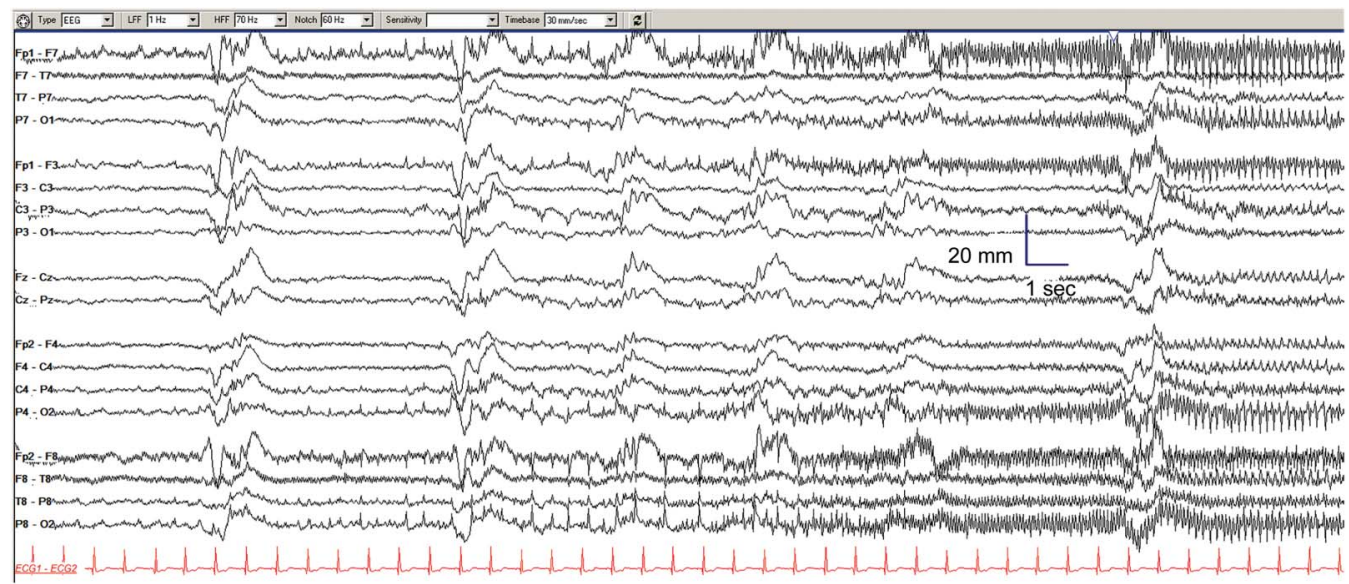

The patient's EEG while she was in the neuroscience intensive care unit. There is a discontinuous (burstsuppression) background at baseline. A seizure arises with a complex field involving the right temporal and parietal regions but also the left frontal region, then evolving to become diffuse. 
if not detected early. ${ }^{2,5}$ Because status epilepticus and dysautonomia are common, this syndrome is best managed by a neurointensivist and epileptologist along with a dermatologist. DNS recurrence is rare. In a reported case of recurrence, the patient died of the disease. ${ }^{6}$

Treatment for DNS is largely empiric, but IVIg has been shown to be well-tolerated and of some benefit, with maintenance therapy required to prevent recurrence. ${ }^{2,5}$ Since the patient had a prior episode that was successfully treated, her previous regimen of steroids, plasmapheresis, and IVIg was repeated, leading to resolution.

The pathogenesis of DNS is unknown. ${ }^{1,2,5,6}$ Brain autopsies from patients with DNS revealed varying and inconclusive results. ${ }^{5}$ Common features of DNS are gliosis and demyelination. ${ }^{5}$ It is hypothesized that circulating cytokines such as interleukin-1, tumor necrosis factor, and transforming growth factor $-\beta$ stimulate glycosaminoglycan synthesis and fibroblast proliferation in the skin. ${ }^{1,5}$ Further studies assessing genetic aberrations in the immune system and levels of IgG, cytokine, and interleukin during an episode of DNS may provide further insight into the pathomechanism of this syndrome.

\section{REFERENCES}

1. Bolognia JL, Jorizzo JL, Schaffer JV. Dermatology, third edition. Philadelphia: Elsevier Saunders; 2013: 687-698.

2. Fleming KE, Virmani D, Sutton E, et al. Scleromyxedema and the dermato-neuro syndrome: case report and review of the literature. J Cutan Pathol 2012;39:508-517.

3. Bhoyrul B, Mughal AA, Paulus J, Salamat A, Howarth S. Does dermatoneuro syndrome have a viral aetiology? Clin Exp Dermatol Epub 2015 Jul 14.

4. Wigley F, Friday RP, Shepard JO, Nazarian RM. Case records of the Massachusetts General Hospital: case 8-2015: a 68-year-old man with multiple myeloma, skin tightness, arthralgias, and edema. N Engl J Med 2015;372:1056-1067.

5. Rogioletti F, Merlo G, Cinotti E, et al. Scleromyxedema: a multicenter study of characteristics, comorbidities, course and therapy in 30 patients. J Am Acad Dermatol 2013;69:66-72.

6. Gonzalez J, Palangio M, Schwartz J, Klainer A, Bisaccia E. Scleromyxedema with dermato-neuro syndrome. J Am Acad Dermatol 2000;42:927-928.

Received September 1, 2015. Accepted in final form November 10, 2015.

\section{AUTHOR CONTRIBUTIONS}

A. Liu: acquisition of data, wrote and revised manuscript for intellectual content. K. Suozzi: acquisition of data, wrote and revised manuscript for intellectual content. D. Y. Hwang: acquisition of data, wrote and revised manuscript for intellectual content. J.J. Moeller: acquisition and interpretation of EEG data, revised manuscript for intellectual content. R. Lazova: acquisition and interpretation of dermatopathologic figures, revised manuscript for intellectual content. D. DiCapua: acquisition of data, revised manuscript for intellectual content.

\section{ACKNOWLEDGMENT}

The authors thank Sarah Buckingham, the senior resident supervisor during the patient's hospital course.

\section{STUDY FUNDING}

No targeted funding reported.

\section{DISCLOSURES}

A.J. Liu and K. Suozzi report no disclosures. D.Y. Hwang receives support from the NIH/NIA Loan Repayment Program, American Brain Foundation, Apple Pickers Foundation, and Neurocritical Care Society. J.J. Moeller has received funding for travel or speaker honoraria from QuantiaMD and NeuroSeriesLive and receives research support from UpToDate, Inc. and Compendium of Therapeutic Choices (Canada). R. Lazova reports no disclosures. D.B. DiCapua serves on a scientific advisory board for the Myasthenia Gravis Foundation of America, serves on the Prime Therapeutics P\&T committee, and attends quarterly meetings, for which he is reimbursed travel expenses and receives honoraria. Full disclosure form information provided by the authors is available with the full text of this article at Neurology.org/cp. 